\title{
EFFECT OF DIETARY RESTRICTION ON OVARIAN COMPENSATORY HYPERTROPHY IN THE RAT
}

\author{
K. J. COOPER* \\ University of Nottingham School of Agriculture, \\ Sutton Bonington, Loughborough, Leicestershire
}

(Received 24th April 1973)

It is established that food deprivation has a marked adverse effect on vaginal cytology and ovarian weight in the rat. This is generally attributed to an impaired secretion of pituitary gonadotrophins (Piacsek \& Meites, 1967; Howland, 1971a; Negro-Vilar, Dickerman \& Meites, 1971). Recently, Howland (1971b, c, 1972) has examined the ability of the underfed rat to exhibit the compensatory ovarian hypertrophy following unilateral ovariectomy that is known to occur in the fully fed animal (Benson; Sorrentino \& Evans, 1969). He concluded that compensatory hypertrophy was not evident in rats which had been subjected to a period of severe dietary restriction. Further, the report of Howland (1971b) indicates that, although hypertrophy of the remaining ovary may not result from hemicastration, the gonadal atrophy usually associated with food deprivation can be prevented by unilateral ovariectomy.

The influence of a reduced food allowance on the compensatory response was examined by Howland (1971b, c) at only one feed level. A mild level of undernutrition has been shown to be relatively ineffective in causing cyclic irregularities when compared to a period of severe food deprivation (Cooper, Haynes \& Lamming, 1970). The extent to which ovarian compensation is affected by a lowered food allowance may, therefore, be dependent on the degree of dietary restriction imposed.

The object of the experiment described in this communication was to examine the effect of food deprivation on ovarian compensatory hypertrophy in the rat at several feed levels and to determine the point at which hypertrophy ceased to occur.

Virgin rats selected from a colony of Wistar origin were used. The animals were 16 to 18 weeks of age at the start of the experiment and were housed in individual metal cages under a $12 \mathrm{hr}$ light/12 hr dark regimen at a temperature of $21 \pm 2^{\circ} \mathrm{C}$. All animals had free access to water and were fed a standard compounded ration at 14.00 hours each day. Vaginal smears were examined daily and animals were selected for the experiment after the completion of at least two consecutive 4- or 5-day oestrous cycles. Eighty rats were randomly allocated to treatment groups as follows: twenty rats were allowed unrestricted access to food and the remaining sixty were allocated to three equal groups and fed at restricted feed levels of $75 \%, 62.5 \%$ and $50 \%$ of the mean daily food

* Present address: Department of Physiology, University of Texas, 5323 Harry Hines Blvd., Dallas, Texas 75235, U.S.A. 
consumption of the unrestricted control group. After 14 days of controlled feeding, the mean body weight of each treatment group was calculated. Of the twenty animals allocated to each group, five were killed after 14 days and both the ovaries and uterus were removed, cleaned, blotted dry and weighed. A further five animals were subjected to sham ovariectomy and retained as intact controls. The remaining ten animals were hemicastrated and together with the five control animals were allowed to continue for a second period of 14 days at the designated feed level. For the ten animals which were unilaterally ovariectomized, both ovaries were exposed but only one was removed and weighed, the gonad being taken alternately from the left and right sides. After 28 days, all animals were killed, the body weight was recorded and the ovaries and uteri were cleaned, blotted dry and weighed. Statistical evaluation of the differences between means was by analysis of variance.

The effect of these treatments on mean body, ovarian and uterine weights is summarized in Table 1.

Table 1. Effect of dietary restriction on ovarian compensatory hypertrophy in rats

\begin{tabular}{l|cc|cc|cc}
\hline \multirow{2}{*}{ Feed level } & \multicolumn{3}{|c|}{ Mean body $w$ (g) } & \multicolumn{2}{c|}{ Mean ovary wt (mg) } & \multicolumn{2}{c}{ Mean uterine wt (mg) } \\
& 14 days & 28 days & 14 days & 28 days & 14 days & 28 days \\
\hline Unrestricted (I) & $155 \pm 9 \cdot 6$ & $162 \pm 8 \cdot 7$ & $30 \cdot 8 \pm 4 \cdot 3$ & $31 \cdot 3 \pm 4 \cdot 1$ & $271 \pm 27 \cdot 3$ & $282 \pm 19 \cdot 4$ \\
Unrestricted(O) & $153 \pm 11 \cdot 2$ & $165 \pm 9 \cdot 1$ & $29 \cdot 2 \pm 3 \cdot 8$ & $43 \cdot 1 \pm 4 \cdot 2$ & & $246 \pm 29 \cdot 1$ \\
$75 \%$ (I) & $148 \pm 7 \cdot 8$ & $144 \pm 8 \cdot 9$ & $28 \cdot 3 \pm 3 \cdot 7$ & $26 \cdot 2 \pm 3 \cdot 3$ & $234 \pm 23 \cdot 6$ & $226 \pm 21 \cdot 1$ \\
$75 \%$ (O) & $146 \pm 10 \cdot 0$ & $145 \pm 11 \cdot 1$ & $26 \cdot 9 \pm 3 \cdot 1$ & $36 \cdot 8 \pm 4 \cdot 3$ & & $208 \pm 18 \cdot 9$ \\
$62 \cdot 5 \%$ (I) & $139 \pm 7 \cdot 7$ & $128 \pm 10 \cdot 4$ & $24 \cdot 4 \pm 3 \cdot 8$ & $20 \cdot 8 \pm 3 \cdot 3$ & $179 \pm 20 \cdot 2$ & $154 \pm 17 \cdot 3$ \\
$62 \cdot 5 \%$ (O) & $145 \pm 8 \cdot 2$ & $131 \pm 9 \cdot 3$ & $26 \cdot 1 \pm 2 \cdot 9$ & $29 \cdot 4 \pm 3 \cdot 7$ & & $169 \pm 16 \cdot 4$ \\
$50 \%$ (I) & $130 \pm 12 \cdot 1$ & $124 \pm 9 \cdot 1$ & $22 \cdot 3 \pm 2 \cdot 4$ & $16 \cdot 6 \pm 2 \cdot 0$ & $161 \pm 17 \cdot 7$ & $139 \pm 15 \cdot 6$ \\
$50 \%$ (O) & $133 \pm 8 \cdot 7$ & $119 \pm 8 \cdot 2$ & $23 \cdot 7 \pm 2 \cdot 2$ & $24 \cdot 4 \pm 2 \cdot 2$ & & $121 \pm 14 \cdot 4$ \\
& & & & & & \\
\hline
\end{tabular}

(I) indicates intact animals killed after 14 days or subjected to sham ovariectomy after 14 days and killed at 28 days. (O) indicates animals unilaterally ovariectomized after 14 days and killed at 28 days.

For the intact animals at $\mathbf{2 8}$ days, there was a significant adverse effect of reduced feed level on body weight, ovarian and uterine weight at the $62.5 \%$ and $50 \%$ but not at the $75 \%$ degrees of dietary restriction $(P<0 \cdot 01)$. A significant hypertrophy of the ovary remaining in the animal was observed in hemicastrated rats at the unrestricted and $75 \%$ feed levels when compared both to the weight of the contralateral gonads removed 14 days previously and to the weight of the ovaries from intact animals at 28 days $(P<0.05)$. At the more extreme degrees of food deprivation, a similar increase in ovarian weight from 14 to 28 days of underfeeding was not found in unilaterally ovariectomized rats. Hemiovariectomy either tended to prevent $(62.5 \%$ feed level) or significantly alleviated $(50 \%$ feed level) the ovarian atrophy associated with severe undernutrition $(P<0 \cdot 05)$. There was no alteration in mean uterine weight which could be attributed to unilateral ovariectomy.

The present findings support the hypothesis that the underfed rat retains the ability to compensate for the removal of one ovary, either by subsequent hypertrophy or reduced gonadal atrophy of the remaining gland. The extent to which this is observed depends on the severity of food restriction to which the 
animal is subjected. A mild degree of dietary restriction does not prevent the hypertrophy response from occurring, but the maximal weight increase found in the fully fed animal is not observed. A further increase in the severity of food restriction to a level at which cyclic behaviour becomes irregular or ceases altogether (Cooper et al., 1970) suppresses the compensatory hypertrophy mechanism to the extent that gonadal weight gain fails to occur. The response to hemicastration, however, is sufficient to halt the gradual atrophy of the ovary found in intact controls. This indicates that a compensatory response mechanism is operating even at quite severe degrees of dietary restriction, allowing the animal to alleviate further gonadal atrophy induced by lack of nutrition. This conclusion is not in accord with the report of Howland (1972), who considered that the ability of the hypothalamic-pituitary complex to respond to a reduction in circulating ovarian steroids as a result of unilateral ovariectomy rapidly disappeared after 10 days of underfeeding. The results presented in this communication indicate that the hypothalamic-pituitary complex during underfeeding is in fact still able to compensate for the removal of one gonad either by a hypersecretion or prevention of a decline in pituitary gonadotrophin release.

\section{REFERENGES}

Benson, B., Sorrentino, S. \& Evans, J. S. (1969) Increase in serum FSH following unilateral ovariectomy in the rat. Endocrinology, 34, 369.

Cooper, K. J., Haynes, N. B. \& Laming, G. E. (1970) Effects of unrestricted feeding during oestrus on reproduction in the underfed female rat. F. Reprod. Fert. 22, 293.

Howland, B. E. (1971a) Effect of restricted feed intake on LH levels in female rats. F. Anim. Sci. 34, 445.

HowLAND, B. E. (1971b) Effect of restricted feed intake on ovarian compensatory hypertrophy in the rat. 7. Anim. Sci. 33, 83.

Howland, B. E. (1971c) Castration hypersecretion of luteinizing hormone but lack of ovarian compensatory hypertrophy in underfed rats. Experientia, 27, 1484.

HowLAND, B. E. (1972) Ovarian weight and ovarian compensatory hypertrophy in the rat as affected by duration of underfeeding. F. Reprod. Fert. 28, 321.

Negro-Vilar, A., Dickerman, E. \& MeIres, J. (1971) Effects of starvation on hypothalamic FSH-RF and pituitary FSH in male rats. Endocrinology, 88, 1246.

Piacsek, B. E. \& Mertes, J. (1967) Reinitiation of gonadotrophin release in underfed rats by constant light or epinephrine. Endocrinology, 91, 535. 